\title{
A short term controlled trial of an adjustable oral appliance for the treatment of mild to moderate obstructive sleep apnoea
}

Kathleen A Ferguson, Takashi Ono, Alan A Lowe, Sulaiman Al-Majed, Leslie L Love, John A Fleetham
Obstructive sleep apnoea (OSA) is a common disorder that can present with various clinical consequences. ${ }^{1}$ Nasal continuous positive airway pressure therapy (nCPAP) is a highly effective treatment for OSA, ${ }^{2}$ but there can be substantial problems with patient acceptance and long term compliance. ${ }^{3}$ The most common surgical treatment for OSA is uvulopalatopharyngoplasty (UPPP) but this approach is limited by its variable success. ${ }^{4}$ Consequently, there is a need for alternative treatments for OSA that are safe, effective, and acceptable. Oral appliances represent a relatively new approach in the management of OSA. ${ }^{5}$ SchmidtNowara and co-workers have reported their experience with a mandibular repositioning appliance in 68 patients with either snoring or OSA. ${ }^{6}$ In the 20 patients with follow up polysomnography the appliance reduced the apnoea and hypopnoea index (AHI) by more than $50 \%$ and improved both arterial oxygen saturation and sleep quality. O'Sullivan and co-workers have recently shown that a mandibular advancement splint decreased AHI to $<20$ /hour in 12 of 17 patients in whom untreated AHI was 20-60 per hour, and in two of nine patients in whom untreated AHI was $>60 /$ hour. ${ }^{7}$ Eveloff and colleagues reported their results with an anterior mandibular positioning appliance in 19 patients with OSA. ${ }^{8}$ Their success rate was $53 \%$ when they defined treatment response as a reduction in AHI to $<10 /$ hour with the appliance.

There are major design differences in the numerous oral appliances that are now available and this may have an impact on their success and compliance rates. A novel oral appliance has been developed with an adjustable hinge that allows the progressive advancement of the mandible to achieve an optimal mandibular position. This anterior mandibular positioner (AMP) allows lateral movement, covers all of the dentition, and provides good overnight retention. The primary objective of this study was to compare subjective and objective efficacy, side effects, patient compliance, and preference between AMP and nCPAP in the treatment of patients with symptomatic mild to moderate OSA.

side effects or compliance.

Conclusions - AMP is an effective treatment in some patients with mild to moderate OSA and is associated with greater patient satisfaction than $\mathbf{n C P A P}$.

(Thorax 1997;52:362-368)

Keywords: obstructive sleep apnoea, oral appliances, nasal CPAP.

\section{Methods}

SUBJECTS

Twenty four patients with symptomatic mild to moderate OSA (AHI 15-55/hour of sleep during diagnostic polysomnography) were re- 


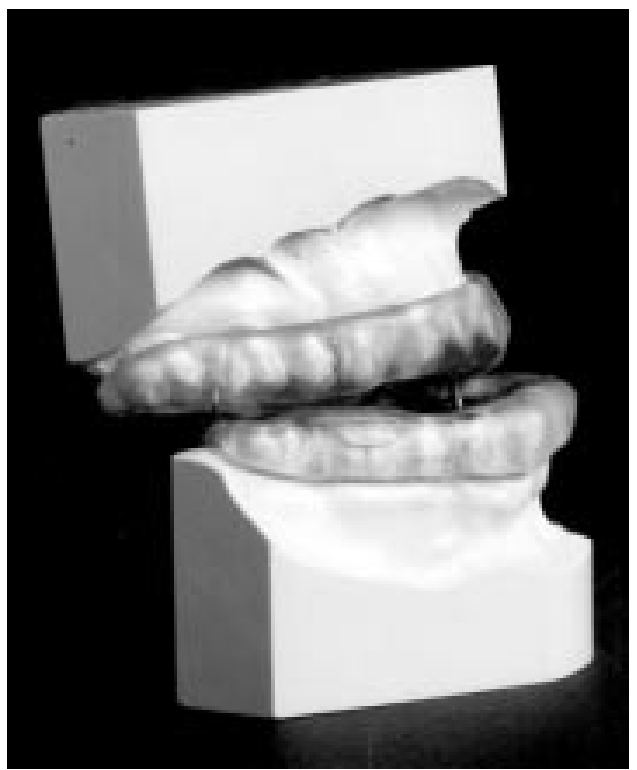

Figure 1 The adjustable anterior mandibular positioner $(A M P)$.

cruited for this prospective crossover study. Patients were unselected apart from a requirement that they had at least 10 teeth in each of the maxillary and mandibular arches, and lived in the metropolitan Vancouver area. All patients were seen in the Sleep Disorders Clinic at the Vancouver Hospital and Health Sciences Centre between February 1993 and April 1994. The patients did not have to pay for either treatment. Each patient gave informed written consent and the study protocol was approved by the clinical screening committee for research and other studies involving human subjects at the University of British Columbia.

\section{TREATMENT}

Oral appliance

The anterior mandibular positioner (AMP) used during this study is a new appliance with several novel features (fig 1). It is constructed of a methyl methacrylate material (SR-Ivocap; Elastomer Ivoclar Co, New York, USA) and the upper and lower portions of the appliance provide full occlusive coverage of the teeth. A titanium hinge with five holes connects the upper and lower portions. This hinge allows a small amount of lateral movement of the jaw. There is a space between the teeth to permit oral airflow. The amount of the mandibular advancement was initially set at $70 \%$ of maximal mandibular advancement. The AMP was adjusted to maximise comfort by relieving pressure points on the teeth and gums. The amount of mandibular advancement was then progressively increased over the next three months by a mean (SD) of $1.8(1.2) \mathrm{mm}$ until snoring ceased and symptoms improved (11 patients), or until the patient could not tolerate further advancement (nine patients). $n C P A P$

nCPAP therapy was undertaken with either a REMstar Choice machine (Respironics Inc, Murrysville, Pennsylvania, USA) or a Tranquillity Plus machine (Healthdyne Technologies, Marietta, Georgia, USA) which were the most advanced nCPAP units available at the time of the study. Patients used various different airway access devices based on their own preference. The use of a cold flow-by humidifier was optional although encouraged. Intranasal corticosteroids and/or anticholinergic medications were used to relieve any nasal symptoms caused by the nCPAP. All patients underwent overnight polysomnography at the beginning of the nCPAP treatment period to determine the optimal pressure necessary to completely relieve the OSA.

QUESTIONNAIRE AND PHYSICAL EXAMINATION All patients underwent a complete history and physical examination before recruitment to the study. A detailed questionnaire that included questions about symptoms, treatment efficacy, side effects, and patient satisfaction was administered during the wash-in and wash-out period and at the end of each treatment period. The Epworth sleepiness scale questionnaire ${ }^{9}$ was also administered at these times. Side effects were rated both in terms of frequency (never, rarely, sometimes, often) and severity (absent, mild, moderate, severe). Patient satisfaction was rated either very satisfied, moderately satisfied, moderately dissatisfied, or very dissatisfied. At the end of the study each patient was asked whether they would prefer to use AMP or nCPAP as a long term treatment.

DIAGNOSTIC POLYSOMNOGRAPHY

Each patient underwent overnight polysomnography before recruitment to the study. Sleep and its stages were documented by standard electroencephalographic (EEG), electrooculographic (EOG), and electromyographic (EMG) criteria. ${ }^{12}$ The EEG was recorded with electrodes applied at C3-A2 and C4-A1 (according to the International 10-20 system) and EMG activity was recorded from the submental muscles. Oronasal airflow was recorded by an infrared $\mathrm{CO}_{2}$ analyser (Model 1260; Novamatrix Medical Systems Inc, Wallingford, Connecticut, USA). A single ECG lead (modified $V_{2}$ ) was monitored to detect cardiac arrhythmias. Oxygen saturation $\left(\mathrm{SaO}_{2}\right)$ was monitored continuously with a pulse oximeter (Model N-100; Nellcor Inc, Hayward, California, USA) attached to the index finger. Chest wall movement was monitored by a respiratory inductive plethysmograph (Respitrace; Ambulatory Monitoring Equipment, Ardsley, New York, USA). The data were recorded on a 15channel polygraph (Model 78; Grass Instruments Co, Quincy, Massachusetts, USA) into the CNS Sleep Lab System (Model 200; CNS, Inc., Chanhassen, Minnesota, USA). All of these studies were manually scored for sleep stage, apnoea type, and duration. Obstructive apnoeas were defined as the cessation of airflow 
for at least 10 seconds accompanied by ongoing respiratory effort, central apnoeas were defined as the cessation of airflow and respiratory effort for at least 10 seconds, mixed apnoeas were defined as a combination of an obstructive and central apnoea lasting for at least 10 seconds, and hypopnoeas were defined as a decrease of more than $50 \%$ in thoracoabdominal amplitude (Respitrace sum) for at least 10 seconds. The severity of OSA was assessed in terms of the AHI.

HOME SLEEP MONITORING

Home sleep monitoring was performed with the Poly-G Portable Apnoea Recorder and Sleep I/T-8 (CNS Inc, Chanhassen, Minnesota, USA). ${ }^{10}$ This combined system recorded EEG (C3-A1), limb EMG, EOG, ECG, oronasal airflow (thermistor), respiratory effort (chest and abdomen), arterial oxygen saturation $\left(\mathrm{SaO}_{2}\right)$, body position, and limb activity. The data were stored and the real time respiratory data were later regenerated for analysis. This allowed full manual analysis of the respiratory data (airflow, respiratory effort, and oxygen saturation). Sleep staging was done by a recently validated automated analysis ${ }^{11}$ as the raw EEG, EOG, and EMG data are not available with this monitoring system.

LATERAL CEPHALOMETRY

Upright lateral cephalometry was performed in all patients before AMP treatment and at the same time as the AMP in one patient who had an increase in AHI with AMP treatment. The analysis of the craniofacial, hyoid position, upper airway size, and soft tissue data has recently been described in detail. ${ }^{12}$

\section{STUDY DESIGN}

The study consisted of a two week wash-in period following the initial diagnostic polysomnogram. During this time the patients were not treated and baseline home sleep monitoring was performed. The questionnaires were administered during the wash-in period. The patients were also weighed at this time and, subsequently, on each occasion the questionnaires were administered. The patients were then randomised to treatment with either AMP or nCPAP. The questionnaires and home sleep monitoring were repeated at the end of the four month treatment period. The patient then had a two week wash-out period during which they were not treated. At the end of this wash-out period the questionnaires were administered and home sleep monitoring was performed. The patient then underwent a second four month treatment period with the other treatment. The questionnaires were administered and home sleep monitoring was performed at the end of this treatment period. Patients were seen monthly during each treatment period. When patients had side effects or were unable to comply with AMP treatment it was re-adjusted to maximise comfort. When patients had side effects or were unable to comply with nCPAP the use of a humidifier was encouraged and the type of airway access device was often changed. Treatment success was defined as a resolution of symptoms and a reduction in $\mathrm{AHI}$ to $<10 /$ hour and treatment failure was defined as ongoing clinical symptoms and/or a reduction in AHI to $>10$ /hour. Compliance failure was defined as an inability or unwillingness of the patient to use the treatment.

STATISTICAL ANALYSIS

The data were analysed according to the method of Jones and Kenward. ${ }^{13}$ Each patient received the treatments in one of two possible orders: sequence A (AMP followed by nCPAP) or sequence $\mathrm{B}$ ( $\mathrm{nCPAP}$ followed by $\mathrm{AMP}$ ). The treatment effect was estimated using the differences of the pairs of observations from each patient (before and after treatment). In order to rule out a treatment-by-period interaction, a test for carryover effect and period effect was performed. ${ }^{14}$ The questionnaire and the home sleep monitoring data were analysed by groups such that the results of nCPAP in sequence A were compared with the results of $\mathrm{nCPAP}$ in sequence $\mathrm{B}$. This was also performed for the AMP treatment from sequences $\mathrm{A}$ and B. Comparisons were also made between the different treatments in each sequence (AMP versus $\mathrm{nCPAP}$ for sequences $\mathrm{A}$ and $\mathrm{B}$ ). The results were pooled if there was no difference between the same treatment from the different sequences and if the differences between the two treatments within each sequence were the same for sequences A and B. The data obtained during the wash-in and wash-out periods before AMP and before nCPAP were compared to ensure that the baseline values preceding each treatment were the same. The questionnaire symptom data were grouped into three possible responses - improved, unchanged, or worse. The home sleep monitoring data were then compared in the same manner as described above before pooling. The symptom, side effect, and compliance data were compared by the Prescott test. The data from AMP sequence A were compared with AMP sequence $B$, and those from nCPAP sequence $A$ were compared with nCPAP sequence $\mathrm{B}$ by an unpaired $t$ test. The results from AMP sequence $A$ were compared with nCPAP sequence $\mathrm{A}$ and those from AMP sequence $B$ were compared with nCPAP sequence $\mathrm{B}$ by a paired $t$ test. The final comparisons were made between treatments with a paired $t$ test on the pooled AMP and nCPAP treatment effect from both treatment sequences. Data that were not normally distributed were log transformed prior to comparison. Single logistic regression analysis was performed to determine whether any clinical, polysomnographic, and cephalometric variables were associated with a successful treatment outcome.

\section{Results}

Twenty four patients (19 men) with symptomatic OSA were recruited to the study. They 
Table 1 Mean (SD) home sleep monitoring data before and with the anterior mandibular position $(A M P)$

\begin{tabular}{lcc}
\hline & Before AMP & \multicolumn{1}{c}{ AMP } \\
\hline AHI* & $25.3(15.0)$ & $14.2(14.7)$ \\
Apnoea index* & $8.2(9.9)$ & $3.6(6.4)$ \\
\% TST supine & $47.1(28.2)$ & $42.3(28.4)$ \\
Desaturations <90\% (no/h) & $13.7(11.7)$ & $12.1(16.9)$ \\
Minimum SaO $_{2}(\%)$ & $78.7(8.6)$ & $75.8(11.6)$ \\
Total sleep time (min) & $390(65.9)$ & $402(72.1)$ \\
Sleep latency (min) & $18.1(10.4)$ & $14.8(11.2)$ \\
Sleep efficiency (\%) & $85.9(5.7)$ & $87.9(5.5)$ \\
NREM (\%) & $83.9(13.2)$ & $85.4(7.1)$ \\
REM (\%) & $16.1(13.6)$ & $13.1(6.0)$ \\
Awakenings (n) & $29.6(12.8)$ & $23.8(12.4)$ \\
\hline
\end{tabular}

TST $=$ total sleep time; $\mathrm{SaO}_{2}=$ arterial oxygen saturation; NREM = non-rapid eye movement sleep; REM = rapid eye movement sleep. * p $<0.005$.

were generally middle aged with a mean (SD) age of 44.0 (10.6) years, overweight (body mass index $\left.32.0(8.2) \mathrm{kg} / \mathrm{m}^{2}\right)$, with mild to moderate OSA (apnoea/hypopnoea index 26.8 (11.9)/ hour). One patient dropped out early in the AMP treatment period because he refused to return for follow up and three refused to cross over from the AMP to the CPAP treatment arm (two treatment successes, one treatment failure). All subsequent results are presented on the remaining 20 patients. There was no carry-over effect between the treatment periods and no period effect on the home sleep monitoring data so these data from the AMP and nCPAP were pooled between the two different treatment sequences. There were no significant differences in the home sleep monitoring data measured before treatment with the AMP and those measured before treatment with nCPAP. There was no significant change in body weight during either the AMP or nCPAP treatment periods.

Twenty patients were treated with the AMP and one $(5 \%)$ was considered to be a compliance failure. The remaining 19 patients had follow up home sleep monitoring performed with the AMP. There was no difference in the time spent sleeping in the supine position during the pre-treatment period or during home sleep monitoring with the AMP. The AHI measured at home before treatment was

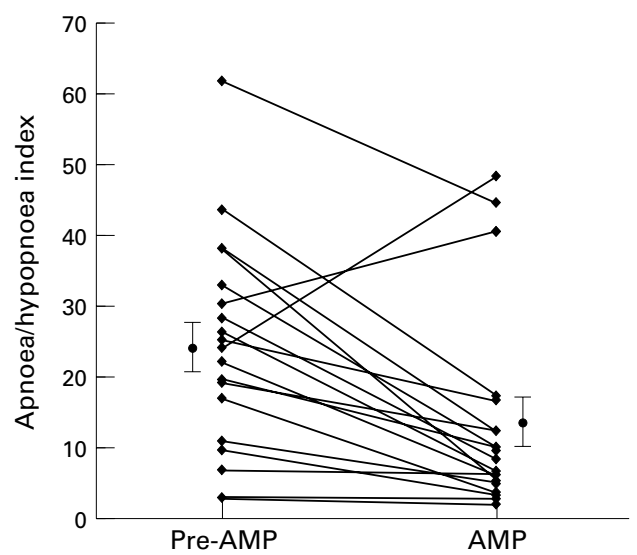

Figure 2 Apnoea/hypopnoea index from home sleep monitoring before and with the anterior mandibular positioner $(A M P)$. The apnoea/hypopnoea index was significantly reduced by the $A M P(p<0.005)$. Values are mean (SE).

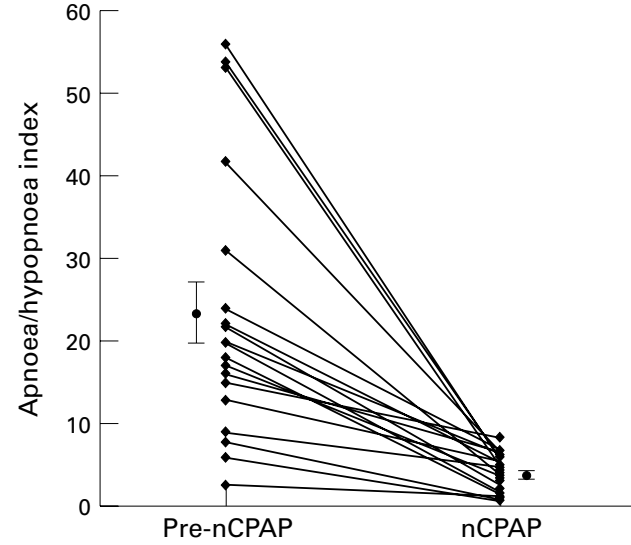

Figure 3 Apnoea/hypopnoea index from home sleep monitoring before and with nasal continuous positive airway pressure (nCPAP). The apnoea/hypopnoea index was significantly reduced by $n C P A P(p<0.005)$. Values are mean (SE).

25.3 (15.0)/hour which decreased to 14.2 (14.7)/hour with the AMP ( $\mathrm{p}<0.005$; table 1 , fig 2). This represents a reduction of approximately $44 \%$ in the AHI with AMP. There were eight treatment failures in whom the AHI was not reduced to $<10 /$ hour (seven patients) or in whom there were ongoing clinical symptoms (one patient). Two of the treatment failures had an increase in AHI with AMP (from 30.7/ hour to 40.6 hour $(32 \%)$ and from 24.3 hour to 48.6 /hour $(100 \%)$ ). The maximum intrasubject variability in AHI between the washin and wash-out periods was 38.5 /hour to 55.5 / hour $(44 \%)$.

Twenty patients used nCPAP and 19 had follow up home sleep monitoring because one of the compliance failures with nCPAP stopped treatment before a follow up home sleep monitoring could be performed. The pre-treatment AHI from home sleep monitoring was 23.5 (16.1)/hour and decreased with nCPAP to 4.2 (2.2)/hour ( $p<0.005$; table 2, fig 3 ). The AHI was lower with nCPAP than with AMP (mean difference 10.2 (14.7)/hour; $\mathrm{p}<0.01,95 \%$ confidence interval 2.9 to 17.5$)$. The number of desaturations was less with nCPAP than with AMP (mean difference 11.1 (18.5)/hour; p $<0.05,95 \%$ confidence intervals 3.6 to 18.6 ) There was no change in sleep architecture or

Table 2 Mean (SD) home sleep monitoring data before and with nasal continuous positive airway pressure $(n C P A P)$

\begin{tabular}{lcc}
\hline & Before $n C P A P$ & $n C P A P$ \\
\hline AHI* $^{*}$ & $23.5(16.5)$ & $4.0(2.2)$ \\
Apnoea index* & $9.0(9.5)$ & $0.7(1.3)$ \\
\% TST supine & $32.5(21.4)$ & $42.7(35.5)$ \\
Desaturations <90\% (no/h)* & $19.4(21.8)$ & $0.4(0.6)$ \\
Minimum SaO $_{2}(\%) *$ & $76.8(9.1)$ & $87.7(2.4)$ \\
TST (min) & $340.4(104.2)$ & $387.9(110.8)$ \\
Sleep latency (min) & $12.8(8.9)$ & $14.6(17.3)$ \\
Sleep efficiency (\%) & $88.4(8.9)$ & $89.8(3.4)$ \\
NREM (\%) & $81.2(16.6)$ & $85.4(8.8)$ \\
REM (\%) & $17.3(15.0)$ & $12.1(5.4)$ \\
Awakenings (n) & $27.1(19.2)$ & $21.1(9.0)$ \\
\hline
\end{tabular}

$\mathrm{TST}=$ total sleep time; $\mathrm{SaO}_{2}=$ arterial oxygen saturation $\mathrm{NREM}=$ non-rapid eye movement sleep; $\mathrm{REM}=$ rapid eye movement sleep. 
Table 3 Symptom response to the anterior mandibular positioner (AMP) and nasal continuous positive airway pressure ( $n C P A P)$

\begin{tabular}{|c|c|c|c|c|}
\hline & \multicolumn{2}{|l|}{$A M P$} & \multicolumn{2}{|l|}{$n C P A P$} \\
\hline & Improved & Unchanged & Improved & Unchanged \\
\hline Moderate to loud snoring + & $55 \%$ & $45 \%$ & $100 \%$ & 0 \\
\hline Witnessed apnoeas & $70 \%$ & $30 \%$ & $70 \%$ & $30 \%$ \\
\hline Unrefreshing sleep & $75 \%$ & $25 \%$ & $45 \%$ & $55 \%$ \\
\hline Excessive daytime sleepiness & $65 \%$ & $35 \%$ & $55 \%$ & $45 \%$ \\
\hline
\end{tabular}

$t \mathrm{p}<0.05$ between AMP and nCPAP.

sleep efficiency with either AMP or nCPAP (table 2).

Both the AMP and nCPAP were effective in reducing symptoms associated with OSA (table 3). Snoring was still present in $45 \%$ of patients with the AMP but was absent in all patients with nCPAP. Excessive daytime sleepiness, as measured by both symptoms and the Epworth sleepiness scale, was improved with both treatments. The Epworth sleepiness scale decreased with AMP from 10.3 (3.1) to 4.7 (2.6) (p $<0.005)$ and with nCPAP from 11.0 (3.8) to 5.1 (3.3) $(\mathrm{p}<0.05)$, but the decrease was not different between the two treatments.

Mild side effects were common with AMP, particularly in the first month of treatment.
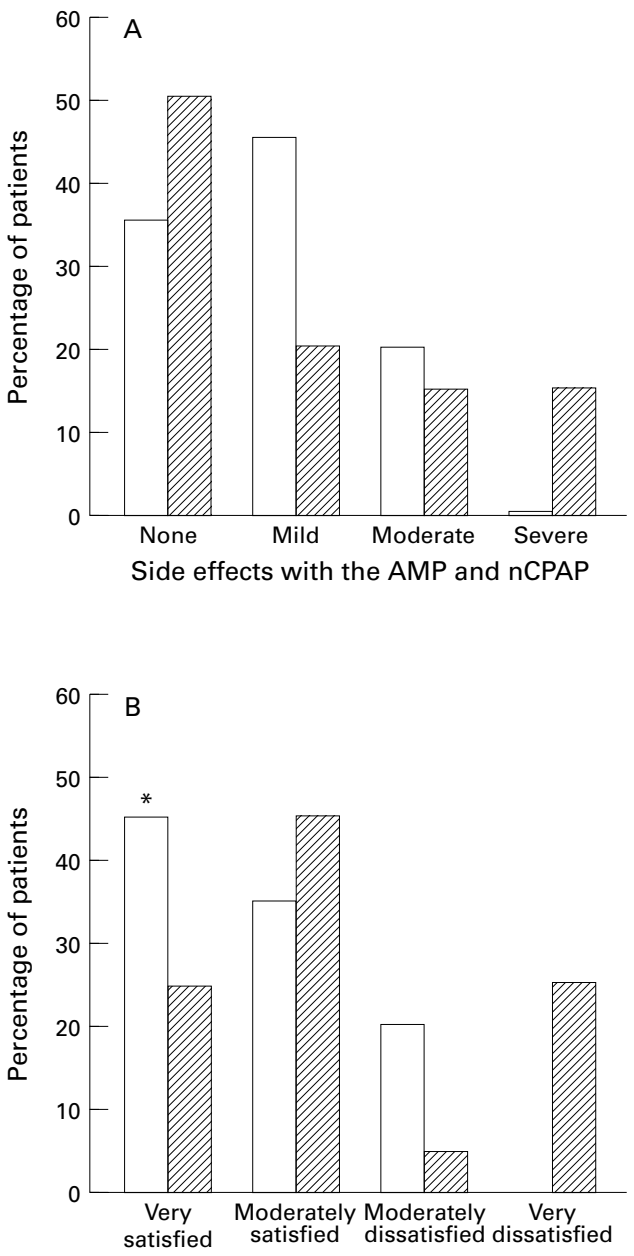

Satisfaction with the AMP and nCPAP
These included sore teeth, sore jaw muscles, excessive salivation, and difficulty chewing in the morning. In most patients the side effects were mild and improved with time. At the end of the four month treatment period nine patients $(45 \%)$ had persistent mild side effects and four $(20 \%)$ had moderate side effects (fig 4). No patient developed any evidence of temporomandibular joint dysfunction. Side effects from nCPAP included nasal congestion, rhinorrhea, eye irritation, and a sense of suffocation. At the end of the four months four patients $(20 \%)$ treated with nCPAP had mild side effects, three $(15 \%)$ had moderate side effects, and three (15\%) had severe side effects. Three patients discontinued nCPAP treatment early either because of side effects, machine noise, or the inconvenience of using nCPAP. The patient who did not have follow up home sleep monitoring with nCPAP discontinued using it because of severe nasal symptoms.

There was no significant difference in side effects between the treatments but the patients were less satisfied with nCPAP $(\mathrm{p}<0.01)$. Sixteen patients $(80 \%)$ were moderately or very satisfied with the AMP and 14 patients (70\%) were very or moderately satisfied with nCPAP.
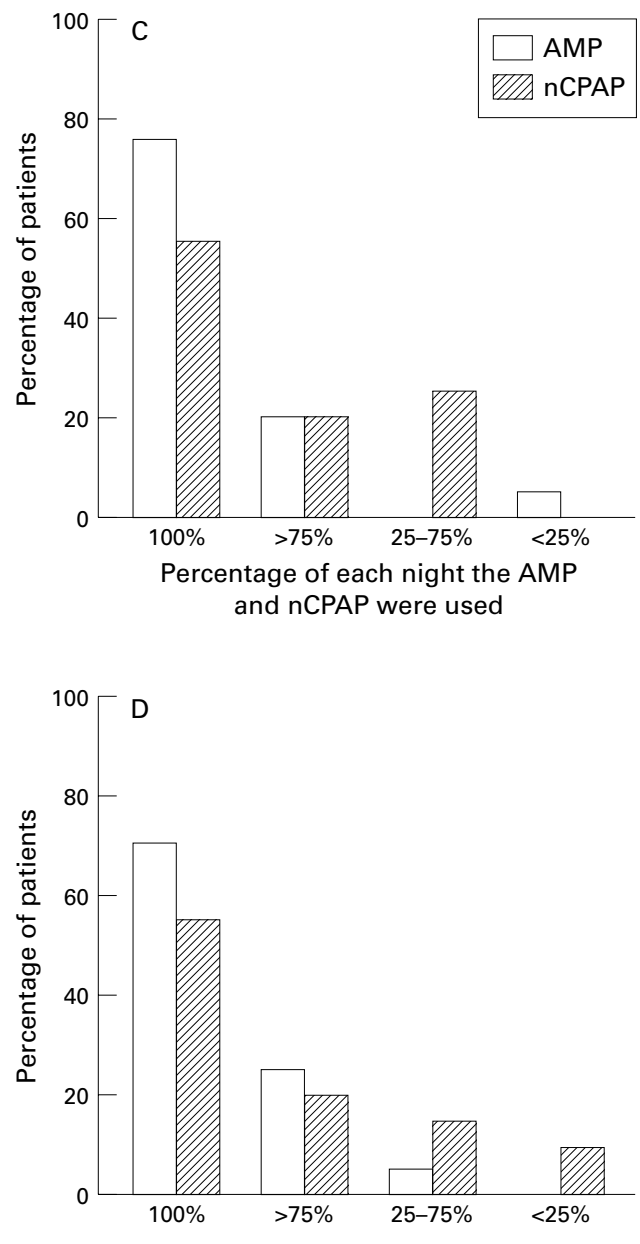

Percentage of nights per week the AMP and nCPAP were used

Figure 4 (A) Side effects, (B) satisfaction, (C) nightly and (D) weekly compliance with the anterior mandibular positioner $(A M P)$ and nasal continuous positive airway pressure $(n C P A P) .{ }^{*} p<0.01$. 


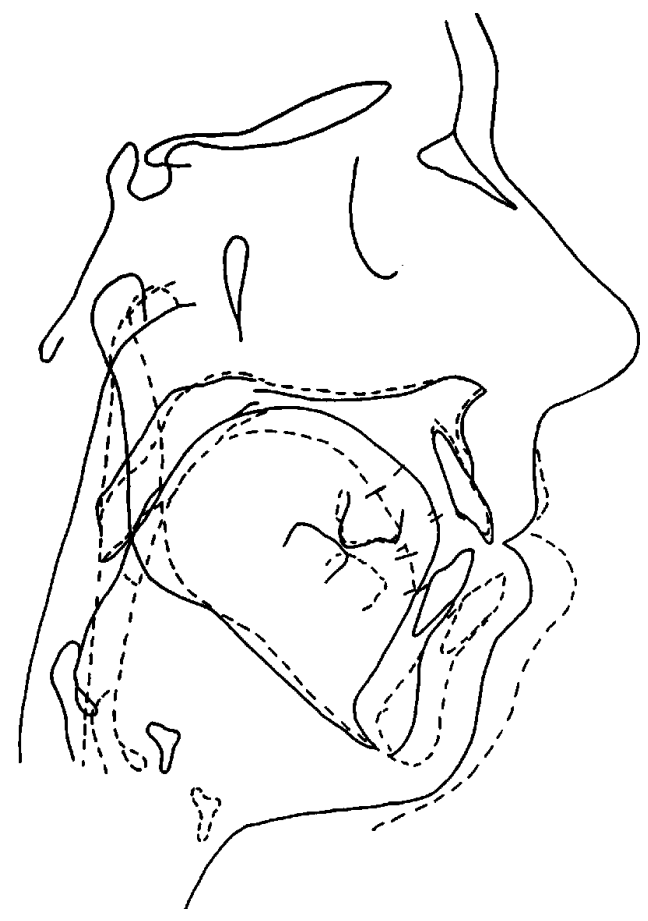

Figure 5 Upright lateral cephalometry without (solid line) and with AMP (broken line) in the patient with a $100 \%$ increase in apnoea/hypopnoea index with AMP.

At the end of the study 10 of the 11 patients who were treatment successes with AMP preferred it to $\mathrm{nCPAP}$ and one preferred $\mathrm{nCPAP}$ to AMP as a long term treatment. Five of the remaining patients preferred nCPAP, one patient used a different oral appliance, one patient proceeded with uvulopalatopharyngoplasty, and two elected to continue with AMP therapy despite an AHI of $>10 /$ hour because their symptoms were relieved and they were unable to tolerate nCPAP. Eight patients were treatment successes with both treatments, seven of whom preferred AMP and one preferred nCPAP as a long term treatment. Patients cited improved comfort, lack of noise, and portability as reasons for this preference. Compliance was assessed subjectively by patient questionnaire data (fig 4). There was no difference in the reported percentage of the night or nights per week treatment was used between the AMP and nCPAP groups. Based on our pre-study outcome definitions, 11 of the $20(55 \%)$ were treatment successes, one $(5 \%)$ was a compliance failure, and eight $(40 \%)$ were treatment failures in the AMP group. In the nCPAP group $14(70 \%)$ were treatment successes, six $(30 \%)$ were compliance failures, and there were no treatment failures.

There were no clinical, polysomnographic, or cephalometric variables predictive of a successful treatment outcome with either AMP or nCPAP. Cephalometry data are presented in fig 5 with and without AMP in the patient who had a large increase in AHI with AMP. AMP caused a decrease in upper airway size associated with posterior movement of the tongue and inferior movement of the hyoid bone.
Discussion

This study is one of the first prospective clinical studies to compare an oral appliance with nCPAP in the treatment of an unselected group of patients with mild to moderate OSA. AMP was found to be an effective treatment in some of these patients with a success rate of $55 \%$ in the AMP group and $70 \%$ in the nCPAP group. However, nCPAP was more effective at improving both AHI and sleep arterial oxygen desaturation. Both treatments improved symptoms and subjective daytime sleepiness. There was no difference in reported compliance or side effects between the treatments but the AMP was associated with greater patient satisfaction than nCPAP. The long term preference was overwhelmingly in favour of AMP therapy with 10 of the 11 patients who were successfully treated with the AMP preferring it as their long term treatment option. Only six patients in the study preferred nCPAP as their long term treatment option.

Our study provides no insights into which patients are most likely to achieve a successful treatment outcome with an oral appliance. Previous studies have suggested that the tongue retaining device is more successful in nonobese patients in whom the OSA is more severe in the supine position. ${ }^{15}$ It has also been proposed that patients with milder OSA are more likely to benefit from mandibular repositioning appliances. ${ }^{6}$ Eveloff and colleagues performed lateral cephalometry in patients with OSA with and without a mandibular repositioning appliance and compared the responders and nonresponders. ${ }^{8}$ They found that a shorter soft palate and a decreased distance between the hyoid bone and the mandibular plane were both associated with a successful treatment outcome. An important finding in our study is that two patients developed more severe OSA with AMP treatment. We do not believe that this increase in the severity of OSA with AMP is explained by intrasubject variability as in at least one of these two patients the increase in AHI was much greater than the maximum intrasubject variability observed in between the wash-in and wash-out periods. This highlights the importance of follow up polysomnography in all patients treated with oral appliances to assess the efficacy of the treatment objectively. The proposed mechanism of action of AMP is that it increases upper airway size by advancing the mandible and tongue. ${ }^{5}$ However, in at least one of our patients the AMP increased the vertical space between the upper and lower incisors causing a downward rotation of the mandible and a decrease in upper airway size associated with an increase in the severity of the OSA.

Our study has certain limitations. The sample size was relatively small and not all patients completed the study. One patient dropped out and another three refused to cross over to nCPAP after using the AMP. One compliance failure with the AMP and one with nCPAP did not have follow up home sleep monitoring. Some of these problems are inherent in any clinical trial. Our crossover design has excellent power characteristics for detecting 
small treatment effects and any potentially confounding carry-over or period effects. ${ }^{13}$ We used home sleep monitoring to establish treatment efficacy. This was necessary because of the expense associated with the large number of follow up studies. We believe home sleep monitoring is appropriate for treatment follow up once the diagnosis of OSA has been established. All treatment comparisons were made based on data from the home sleep monitoring and no comparisons were made with the initial diagnostic polysomnography. Two recent studies have validated our home sleep monitoring system and have shown excellent reliability between laboratory and home sleep data. ${ }^{116}$ We also recognise the limitations of subjective reports of treatment compliance, but at the time of the study the technology was not available for covert monitoring of compliance with either oral appliances or nCPAP. Finally, we only present subjective data on excessive daytime sleepiness and recognise that, if the resources had been available, objective data would have been preferable.

nCPAP remains the primary treatment for patients with severe OSA and associated arterial oxygen desaturation and excessive daytime sleepiness. We have shown that AMP therapy is effective in some patients with mild to moderate OSA and is associated with greater patient satisfaction than nCPAP. Current recommendations are that oral appliances are indicated as first line therapy for mild OSA but only for moderate to severe OSA if other treatment modalities fail. ${ }^{17}$ These recommendations are based on data from case series studies rather than randomised controlled clinical trials. ${ }^{18}$ Our results suggest that oral appliances should be considered first line treatment for patients with symptomatic mild to moderate OSA as most prefer this treatment to nCPAP when both treatments have been shown to be effective. Oral appliances can also be used as an adjunct to nCPAP therapy when the patient is away from home and unable or unwilling to use $\mathrm{nCPAP}$. Another area to research is combined treatment with UPPP and an oral appliance in patients in whom UPPP does not completely relieve OSA.

There are currently at least 13 different oral appliances available for the treatment of OSA. ${ }^{5}$ These appliances have been designed to elevate the soft palate, advance the mandible, and/or hold the tongue forward. There are ongoing developments in oral appliance material and design. The AMP was designed to provide progressive mandibular advancement to an optimal position consistent with the maximum relief of OSA and minimal side effects. AMP is associated with improved treatment success
(55\% versus $48 \%$ ) and fewer compliance failures ( $5 \%$ versus $24 \%$ ) compared with a nonadjustable mandibular repositioning appliance when identical outcome definitions are used. ${ }^{19}$ Larger randomised clinical trials are necessary to determine further the precise indications, benefits, and risks of each oral appliance in the treatment of OSA. These studies should include objective assessment of daytime sleepiness and performance, covert compliance monitoring, and long-term follow up.

The authors thank Dr Barry Wiggs for statistical guidance and Ms Bernice Robillard for assistance with preparation of the manuscript. The anterior mandibular positioner was provided for this study by Space Maintainers, Vancouver, BC and the hinge was supplied by Silent Knights Corporation, Vancouver, BC. Nasal CPAP machines were provided by Respironics Inc Murrysville, Pennsylvania, USA (REMstar Choice) and ARS VitalAire, Vancouver, BC (Healthdyne Tranquillity Plus).

The study was supported by a British Columbia Lung Association Research Grant.

1 Ferguson KA, Fleetham JA. Consequences of sleep disordered breathing. Thorax 1995;50:998-1004.

2 Sullivan CE, Issa FG, Berthon-Jones M, Eves L. Reversal of obstructive sleep apnoea by continuous positive airway pressure applied through the nares. Lancet 1981;i:862-5.

3 Engleman HM, Martin SE, Douglas NJ. Compliance with CPAP therapy in patients with the sleep apnoea/hypopnoea syndrome. Thorax 1994;49:263-6.

4 Ryan CF, Lowe AA, Li D, Fleetham JA. Three-dimensional upper airway computed tomography in obstructive sleep apnea: a prospective study in patients treated by uvulopalatopharyngoplasty. Am Rev Respir Dis 1991;144:42832 .

5 Lowe AA. Dental appliances for the treatment of snoring and obstructive sleep apnea. In: Kryger $\mathrm{MH}$, Roth T, Dement WC, eds. Principles and practice of sleep medicine. 2nd ed. Philadelphia: WB Saunders, 1994: 722-35.

6 Schmidt-Nowara WW, Meade TE, Hays MB. Treatment of snoring and obstructive sleep apnea with a dental of snoring and obstructive sleep
orthosis. Chest 1991;99:1378-85.

7 O'Sullivan RA, Hillman DR, Mateljan R, Pantin C, Finucane KE. Mandibular advancement splint: an appliance to treat snoring and obstructive

8 Eveloff SE, Rosenberg CL, Carlisle CC, Millman RP. Efficacy of a Herbst mandibular advancement device in obstructive sleep apnea. Am F Respir Crit Care Med 1994; 149:905-9.

9 Johns MW. A new method for measuring daytime sleepiness: the Epworth sleepiness scale. Sleep 1991;14:540-5.

10 Man GCW, Kang BV. Validation of a portable sleep apnea monitoring device. Chest 1995;108:388-93.

11 Orr WC, Eiken T, Petram V, Jones R, Rundell OH. A laboratory validation of a portable system for remote recording of sleep-related respiratory disorders. Chest 1994;105:160-2.

12 Ferguson KA, Ono T, Lowe AA, Ryan CF, Fleetham JA. The relationship between obesity and craniofacial structure in obstructive sleep apnea. Chest 1995;108:375-81.

13 Jones B, Kenward M. Design and analysis of cross-over trials. New York: Chapman and Hall, 1989.

14 Everitt B. Statistical methods for medical investigations. New York: Oxford University Press, 1989.

15 Cartwright RD. Predicting response to the tongue retaining device for sleep apnea syndrome. Arch Otolaryngol 1985; 111:385-8.

16 Biernacka H, Douglas NJ. Evaluation of a computerised polysomnography system. Thorax 1993;48:280-3.

17 Standards of Practice Committee of the American Sleep Disorders Association. Practice parameters for the treatment of snoring and obstructive sleep apnea with oral appliances. Sleep 1995;18:511-3.

18 Schmidt-Nowara W, Lowe A, Wiegand L, Cartwright R, Perez-Guerra F, Menn S. Oral appliances for the treatment of snoring and obstructive sleep apnea: a review. Sleep 1995;18:501-10.

19 Ferguson KA, Ono T, Lowe AA, Keenan SP, Fleetham JA. A randomized crossover study of an oral appliance vs. nasal continuous positive airway pressure in the treatment of mild-moderate obstructive sleep apnea. Chest 1996; 109:1269-75. 